Chapter 5

\title{
Nuclear Receptor Modulators - Current Approaches and Future Perspectives
}

\author{
Thales Kronenberger, Oliver Keminer, \\ Carsten Wrenger and Björn Windshügel \\ Additional information is available at the end of the chapter \\ http://dx.doi.org/10.5772/59666
}

\section{Introduction}

In multicellular organisms the regulation of growth, development and metabolic homeostasis involves extensive intercellular communication. This is achieved by diverse endocrine signal molecules that often address intracellular receptors which regulate gene expression in a liganddependent manner. Proteins involved in up- or down-regulation of gene expression are termed transcription factors. It is estimated that about $10 \%$ of the human genome encodes proteins of this family [1]. An important class of transcription factors are nuclear receptors (NRs). So far, 48 different NRs have been identified in humans. However, due to alternative splicing the number of different functional NRs is substantially larger [2]. Similar to other protein families (e.g. G protein-coupled receptors) a unified nomenclature system has been established in order to overcome problems due to multiple names for the same gene [3].

NRs recognize and bind small molecules that comprise, for example, steroid and thyroid hormones, vitamins as well as fatty acids and their derivatives [4]. In fact, for only about half of human NRs an endogenous ligand has been identified so far. The involvement of several members of the NR superfamily in various diseases has made this class of transcription factors highly attractive for pharmaceutical industry. As described below, several members of the NR family are already addressed by drugs and more receptors are under investigation [5].

Understanding nuclear receptor function requires knowledge of the NR structure. The composition of nuclear receptors is modular and involves 5-6 domains with distinct functions (Figure 1). Evolutionary most conserved domains are the DNA-binding domain (DBD) and the ligand-binding domain (LBD). Other domains show a considerable variation in length and 
sequence such as the N-terminal domain, the hinge region - connecting DBD and LBD - as well as the C-terminal domain.

The N-terminal domain (NTD) possesses a ligand-independent activation function 1 (AF-1) and contains several post-translational modification sites [6, 7]. The NTD size may vary considerably, ranging from 23 to 602 residues. Although no X-ray crystal structures of this domain are available, circular dichroism studies have indicated the presence of some secondary structures upon posttranslational modifications [8]. The DBD domain consists of about 70 highly conserved amino acids and contains two zinc-finger motifs which are essential for DNA binding [9]. The DBD organisation allows binding to specific DNA sequences (responsive elements) [9]. The core region of the response elements is organised as hexameric motif with the consensus sequence 5'-AGGTCA-3' [8]. The number of motifs as well as their organisation and spacer length between the repetitive elements and the flanking regions affect the specificity of NR binding [10,11]. A typical response element presents two repetitions of the core motif that can be organised in inverted, everted or direct repeats [12].
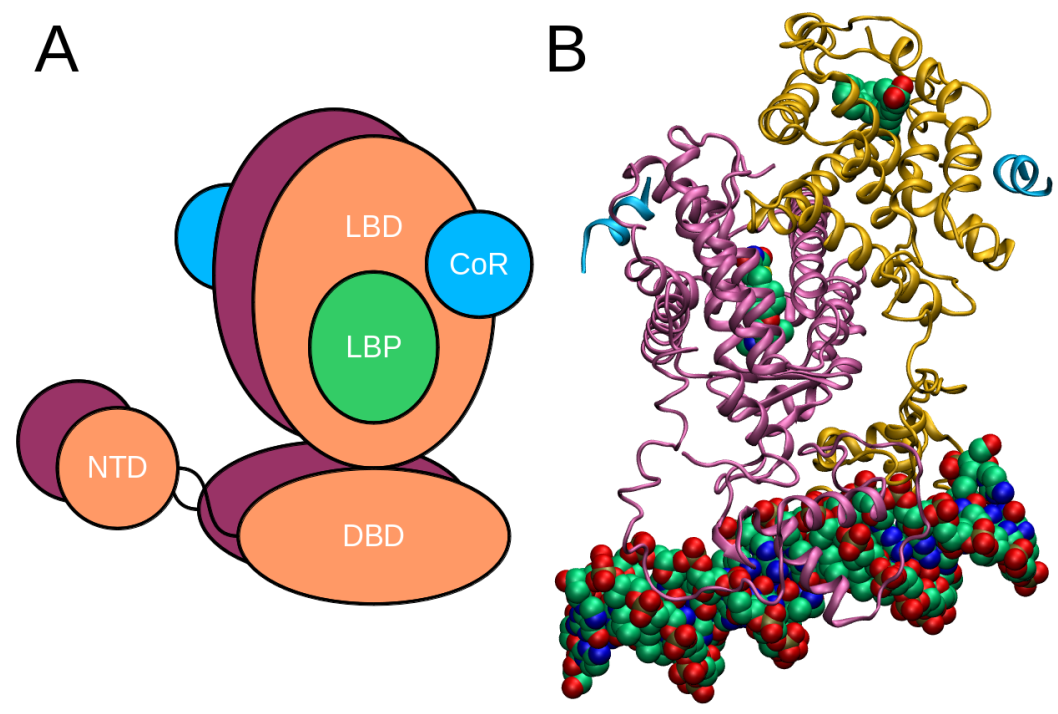

Figure 1. Assembly of nuclear receptor and their interaction partners. A: Nuclear receptors are composed of several domains. Main domains are i) the N-terminal domain (NTD) of variable length that carries the ligand-independent activation function 1 (AF-1), ii) the DNA-binding domain (DBD) that binds to the response elements in the promoter region of target genes and iii) the ligand-binding domain (LBD) that harbours the ligand-binding pocket (LBP), the ligand-dependent activation function (AF-2) as well as a binding site for coregulatory proteins (CoR) and other nuclear receptors.Most nuclear receptors bind as dimers to their response elements. B: X-ray crystal structure (PDB code 3DZY of the PPAR $\gamma$ (violet) and RXR $\alpha$ (orange) DBD and LBD (cartoon representation) bound to DNA (CPK representation). Structure was solved in complex with NR agonists (shown in CPK representation) rosiglitazone (PPAR $\gamma$ ) and 9-cis retinoic acid $(\mathrm{RXR} \alpha)$ as well as coactivator peptides (blue ribbons).

The second large domain is the ligand-binding domain (LBD) that is connected to the DBD via the hinge region. As the name already indicates, the LBD is capable to bind small molecules 
in its ligand-binding pocket (LBP) [12]. In addition, the LBD carries the ligand-dependent activation function 2 (AF-2), located on its C-terminal helix (helix 12, H12) [13]. In addition, the LBD contains a dimerisation motif that allows binding of other NRs and an interaction site for co-regulatory proteins that are important for activation and inhibition of target gene expression [14, 15]. The LBD comprises about 250 amino acids and is mainly composed of $\alpha$ helices, arranged in a so-called three-layered helix sandwich. The ligand-binding pocket is found between both outer layers. Size and amino acid composition of the LBP differ substantially among different NRs resulting in LBP volumes ranging between $100 \AA^{3}$ (ERR $\alpha$ ) and 1300 $\AA^{3}$ (PPAR $\left.\gamma\right)[13,14]$. In some cases, for example NURR1, no ligand-binding pocket is present, suggesting a ligand-independent mechanism of action [23].

Ligand-binding to the LBP modulates the conformation of helix 12 (AF-2). Agonists induce AF-2 to adopt a helical conformation that covers the ligand-binding pocket like a lid. By this process, a binding site for co-activator proteins is generated. These bind to the receptor via their nuclear receptor interacting domain (NRID) which contains a highly conserved LxxLL motif $(\mathrm{L}=$ leucine, $\mathrm{x}=$ any residue) [15]. Several co-activators (e.g. SRC-1) contain an intrinsic histone acetyltransferase function which results in decondensation of the chromatin at the promoter region, thereby improving accessibility of further transcription factors. In addition, co-activators recruit other proteins with histone modifying capabilities as well as proteins of the basal transcription machinery. Eventually, these processes initiate target gene expression. In contrast, NR antagonists displace $\mathrm{H} 12$ from the active conformation, which is often associated with partial unfolding of the helix. This event induces binding of co-repressor proteins to the receptor. Similar to co-activators, further proteins are recruited that lead to chromatin condensation (e.g. by histone deacetylases), thereby silencing gene expression.

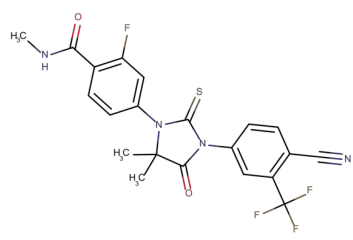

Enzalutamide

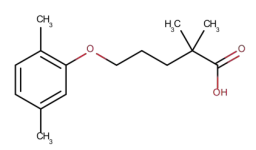

Gemfibrozil

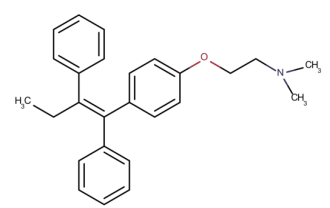

Tamoxifen

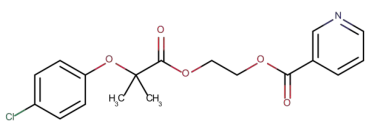

Etofibrate

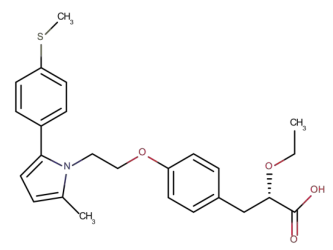

Saroglitazar<smiles>CCOC(=O)C(C)(F)Oc1ccc(Cl)cc1</smiles>

Clofibrate

Figure 2. Examples for approved drugs targeting nuclear receptors. 
Besides other drug target classes such as G protein-coupled receptors, ion channels or receptor tyrosine kinases, nuclear receptors represent another major receptor target class. As of 2011, 76 approved drugs targeting 17 nuclear receptors were available (See Figure 2 for selected examples) of which several generate more than 1 billion dollar sales each year [5]. In this chapter we will highlight selected NRs which are targeted by approved drugs and provide insight into current efforts to address additional receptors using small molecules. A focus will be on novel mechanisms of receptor inhibition as shown by co-activator-binding inhibitors. In addition, currently used methods for studying nuclear receptor function in drug discovery are described.

\section{Pharmaceutically relevant nuclear receptors and their drugs}

Most nuclear receptors addressed by approved drugs belong to the subfamilies 1 (thyroid receptor like receptors) and 3 (estrogen receptor like receptors). Main indication areas are cancer, hormone replacement and metabolic diseases.

An NR that is targeted by both, agonists and antagonists, is the estrogen receptor (ER), belonging to the steroid hormone receptors. Two ER forms exist, called $\alpha$ and $\beta$ (NR3A1, NR3A2). An endogenous ligand of both ERs is the steroid hormone 17 $\beta$-estradiol. While estradiol preferentially binds to the $\alpha$-form (Figure 3A \& C), the third estrogen produced in humans (estriol) favors the $\beta$-form. Both ER $\alpha$ and ER $\beta$ bind as homodimers to their response elements. The natural ligand estrogen is also applied in hormone replacement therapy.

Of main pharmaceutical relevance is ER $\alpha$. In the majority of breast cancers ( 70 \%), ER $\alpha$ is overexpressed in breast tissue (ER $\alpha$-positive cancer). Since the natural ligand estradiol plays an important role in breast cancer development and progression, antagonists targeting ER $\alpha$ have been developed for treatment of ER $\alpha$-positive breast cancer [16]. A frequently used drug that addresses ER $\alpha$ is tamoxifen (Figure 2), a potent antagonist of the receptor. Being a prodrug, tamoxifen requires conversion to the bioactive forms 4-hydroxytamoxifen and N-desmethyl-4hydroxytamoxifen in the liver by cytochromes CYP2D6 and CYP3A4. Both metabolites possess up to 100-fold better affinity to the receptor than the prodrug [17]. Tamoxifen and its metabolites belong to the class of selective estrogen receptor modulators (SERMs), which are chemically different to the natural ligand estradiol. In breast tissue, tamoxifen metabolites act as competitive inhibitors of the natural ligand estradiol in the ER ligand-binding pocket, while in other tissues such as the endometrium, the compounds act as potent ER agonist [18]. This agonistic effect is problematic as it substantially increases the risk of uterine cancer and therefore the compound is not used for long-term treatment [27].

Another selective estrogen receptor modulator is the benzothiophene raloxifene which is applied for treatment and prevention of osteoporosis in postmenopausal women but also for reducing the breast cancer risk. The compound is not a prodrug like tamoxifen as it already contains two hydroxyl groups that form hydrogen bonds with the same LBP-residues as the tamoxifen metabolites. Also a difference is the mechanism of action as raloxifene does not show 


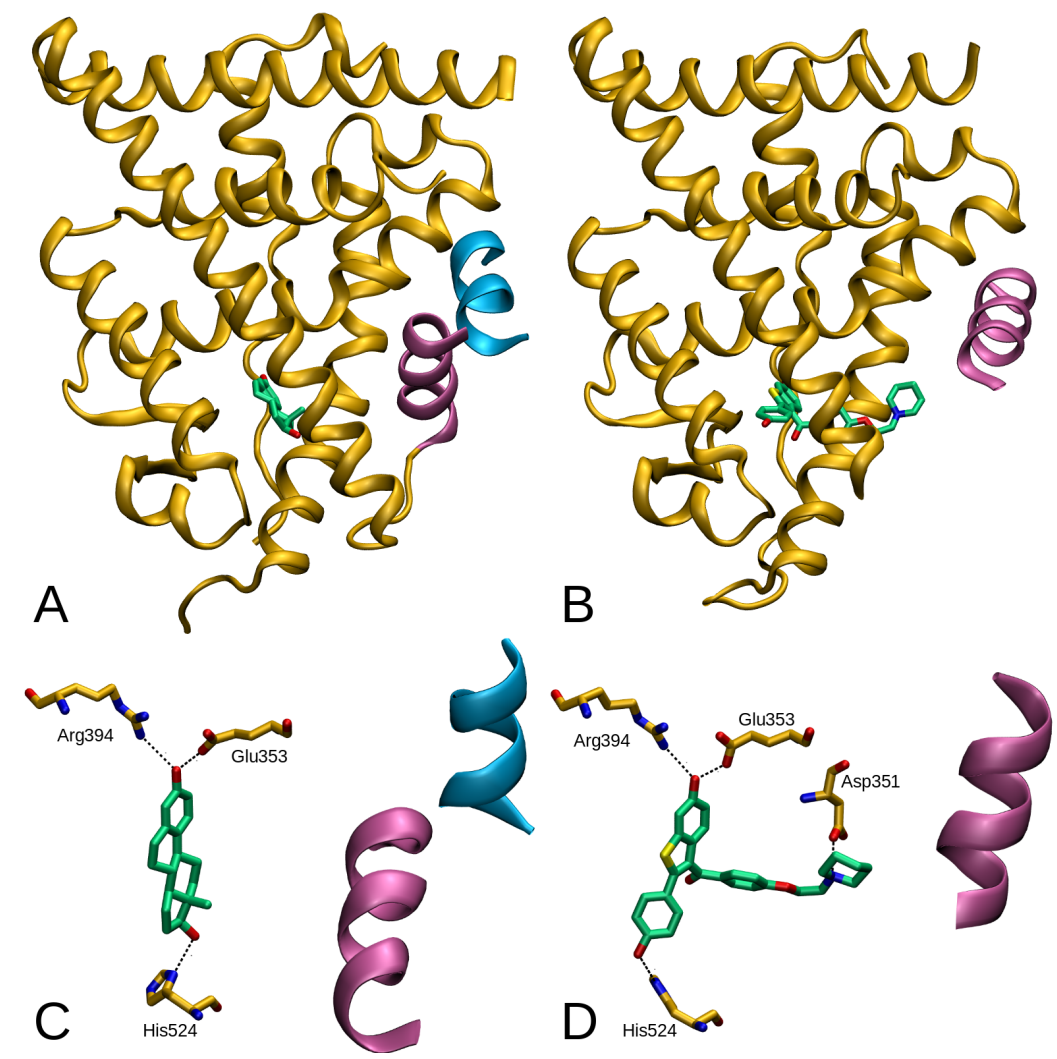

Figure 3. Protein-ligand interactions in estrogen receptor $\alpha$ (ER $\alpha)$. A: ER $\alpha$ (cartoon representation) in complex with the natural agonist estradiol (capped sticks representation, carbon atoms in green, oxygen atoms in red). Helix 12 (violet) is in the active conformation enabling coactivator binding (NRID in blue). B: Antagonist binding (raloxifene) displaces H12 from the active conformation thereby disrupting the coactivator binding site. C \& D: Binding modes of estradiol (C) and raloxifene (D) within the ER $\alpha$ LBP. Both compounds have an identical hydrogen bond interaction pattern (black dotted lines). In addition, raloxifene forms a salt bridge with Asp351.

any antitumor activity. Instead, the compound is used for preventing osteoporosis and may also reduce the incidence of breast cancer in postmenopausal women.

Both SERMs are T-shaped molecules. X-ray crystal structures of estrogen receptor $\alpha$ cocrystallized with 4-hydroxytamoxifen and raloxifene have revealed the binding mode within the LBP (Figure 3B \& D). The core structure of both compounds is planar and binds in a similar orientation into the ligand-binding pocket as the natural ligand estradiol (Figure 3A \& C). Several hydrogen bonds shared with the receptor ensure tight binding. Hydrogen bond formation with the receptor is only possible for the metabolized forms of tamoxifen and explains why these molecules are much more potent compared to the prodrug. The side chain protruding from the core structure of tamoxifen metabolites and raloxifene sterically displaces $\mathrm{H} 12$ from the active conformation, resulting in an inactive NR [25, 26]. 
Another member of the nuclear receptor superfamily targeted by drugs is the androgen receptor (AR, NR3C4). Natural AR ligands are the androgens testosterone or dihydrotestosterone (Figure $4 \mathrm{~A}$ ), both activating the receptor. AR is expressed in several tissues of which the prostate and adrenal gland are representing the main expression sites [19]. Besides its role in sexual differentiation in utero and male pubertal genesis, AR is involved in maintenance of libido, spermatogenesis, muscle mass and strength, bone mineral density and erythropoiesis [20]. Several diseases such as prostate cancer or androgen insensitivity syndrome have been linked to AR [19]. For treatment of prostate cancer hormone deprivation using chemical (luteinizing-hormone-releasing hormone analogues, LHRHa) or surgical castration is a standard therapy that is initially effective in reducing the number of circulating tumor cells. But almost invariably resistance emerges after few years. This type of cancer is then referred to as castration-resistant prostate cancer (CRPC) with poor prognosis. By virtue of AR gene overexpression and amplification as well as mutations within the AR gene, androgen receptor activity is upregulated in CRPC. In order to treat CRPC, AR antagonists, also termed antiandrogens, have been developed.

Both steroidal and non-steroidal AR antagonists have been developed. Low efficacy and hepatotoxicity as well as cardiovascular side effects and problems with libido and potency have limited the use of steroidal antiandrogens. These side effects are largely due to the effect of the drugs on other nuclear receptors recognizing steroid hormones (e.g. progesterone receptor, or glucocorticoid receptor). Non-steroidal anti-androgens (NSAA), which have been introduced about 25 years ago, are mainly used in advanced and metastatic prostate cancer treatment [21]. First generations of NSAAs were flutamides and their derivatives bicalutamide or nilutamide, which are chemically related compounds. The mode of action of these drugs is to compete with the natural ligand for AR binding and thereby antagonizing the receptor and inhibiting tumor growth. While flutamide is usually used in combination with LHRH-a, bicalutamide is also applied as monotherapy. In contrast to steroidal anti-androgens, side effects due to binding to other steroid hormone receptors are less severe.

Enzalutamide (Figure 2), introduced in 2009, is a second generation NSAA used in treatment of CRPC. Besides competing with the natural ligands, the drug also reduces nuclear translocation and, as a consequence, DNA binding of the receptor [22]. Enzalutamide prolongs life of cancer patients, who did not receive chemotherapy before, with only a few registered sideeffects [23]. However, in many CRPC-patients resistance occurs after several months of treatment which had been linked to a mutation within the LBD [24].

In order to overcome resistance problems and to establish therapeutics not targeting the ligandbinding pocket, an alternative approach is to address the protein-DNA interactions of the AR by molecules binding to the DBD. By now several studies have already reported successful identification of compounds targeting DBD of enzalutamide-resistant ARs [25, 26].

Another example for receptors targeted by already approved drugs is the group of peroxisome proliferator-activated receptors (PPARs). Three PPAR subtypes have been identified: PPAR $\alpha$, PPARS (also termed PPAR $\beta$ ) and PPAR $\gamma$. Unlike ER, all PPARs form heterodimers with the retinoid $X$ receptor. Another difference to steroid hormone receptors is a considerably larger LBP. Natural ligands of PPARs include various fatty acids and eicosanoids. Some compounds 


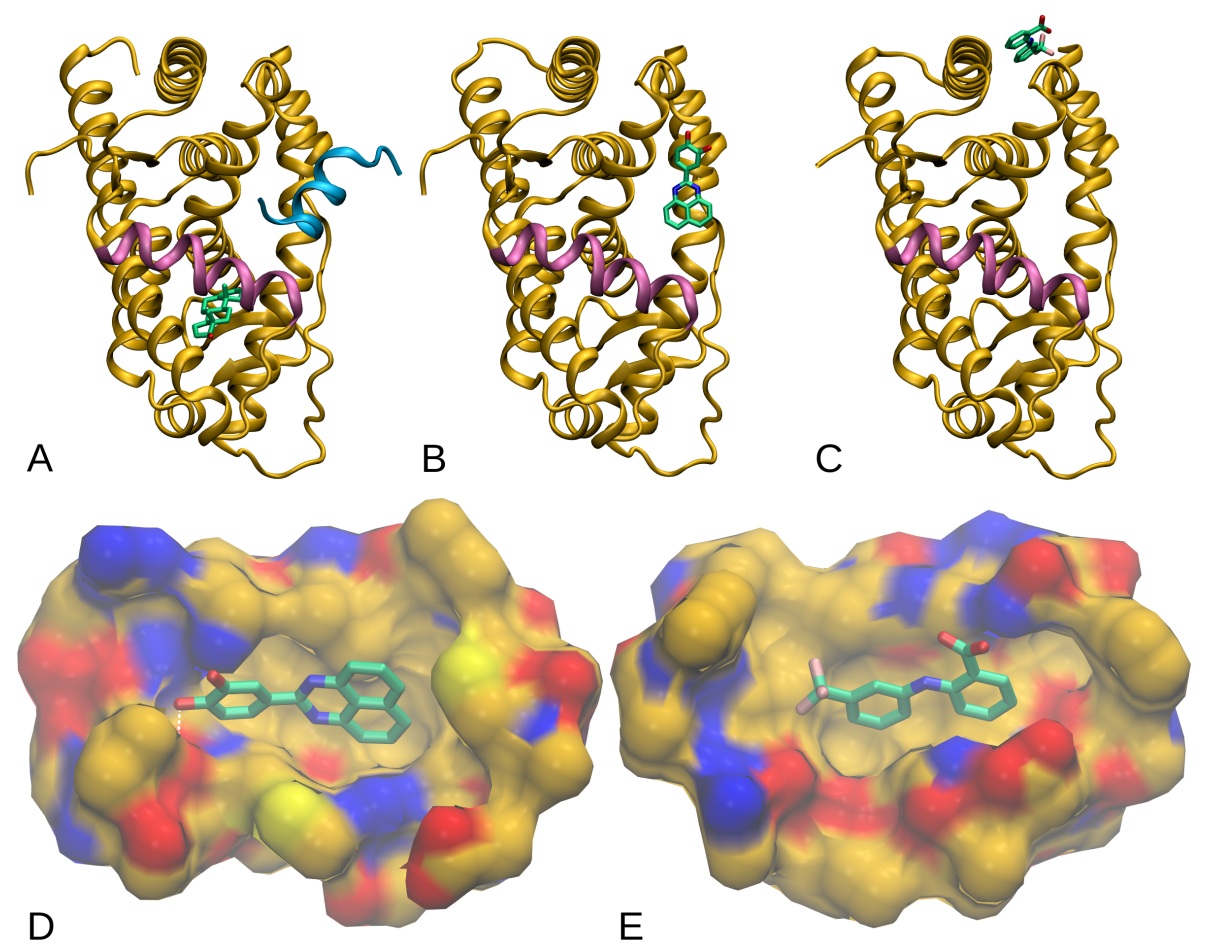

Figure 4. Protein-ligand interactions in androgen receptor (AR). A: AR in complex with testosterone (capped sticks representation) and coactivator peptide (blue). H12 is shown in violet. B: AR in complex with small molecule (AV6) bound to AF-2 site. C: AR in complex with flufenamic acid bound to BF-3 site. D \& E: Binding modes of AV6 (D) and flufenamic acid (E) within AF-2 and BF-3 pockets (shown as surface). AV6 shares a hydrogen bond with the receptor as indicated by the white dotted line.

specifically address single PPAR subtypes. For example leukotriene B4 activates only $\operatorname{PPAR} \alpha$ while a variety of prostaglandins are ligands for PPAR $\gamma$.

All currently approved PPAR drugs target $\alpha$ and $\gamma$ subtypes and are used in treatment of metabolic diseases. PPAR $\alpha$ is addressed by fibrates, e.g. clofibrate or gemfibrozil (Figure 2). Upon receptor activation, a large set of genes is upregulated, including many enzymes involved in lipid metabolism, e.g. lipid transport, oxidation, lipogenesis or cholesterol transport [27]. Compounds of the thiazolidinedione class have been developed for targeting the subtype PPAR $\gamma$ (e.g. rosiglitazone, pioglitazone, troglitazone). Rosiglitazone and pioglitazone are used in treatment of type II diabetes, both activating PPAR $\gamma$ and thereby increasing the sensitivity of adipocytes to insulin which lowers glucose blood levels. Due to liver toxicity, troglitazone has been completely withdrawn from the market.

Another class of PPAR-addressing molecules has been introduced recently. The so-called glitazars are dual PPAR agonists, activating PPAR $\alpha$ and PPAR $\gamma$. In 2013, the first glitazar (saroglitazar) was approved as drug in India while other glitazar research programs have been 
discontinued due to safety reasons. Saroglitazar (Figure 2) is used for treatment of diabetic dyslipidemia and hypertriglyceridemia.

Most nuclear receptors are addressed due to their direct involvement in a disease. However, some members of the NR superfamily are interesting because of their involvement in drug metabolism. This process comprises three phases that involve compound modification (e.g. oxidation by cytochrome P450 enzymes), coupling reactions with hydrophilic substances (e.g. glucuronic acid, glycine) and finally excretion of the metabolised molecules from the cell via transporter proteins. Both, pregnane $X$ receptor (PXR) and the constitutive androstane receptor (CAR), are mainly expressed in liver and intestine and are responsible for liganddependent induction of gene expression of proteins involved in all phases of drug metabolism [50]. Due to their ability to bind structurally and chemically diverse molecules, including many prescription drugs and other synthetic compounds that enter the human body, CAR and PXR are also termed xenosensors [28].

Comparing both receptors, PXR is most promiscuous and recognizes a large set of prescription drugs, among them calcium channel blockers, statins, antidiabetic drugs, HIV protease inhibitors and also artemisinin and its derivatives [29, 30]. PXR X-ray crystal structures have unravelled the basis for the pronounced ligand promiscuity of the receptor. The receptor LBD deviates from the canonical NR fold (H6 unfolded, H7 broken, long H1-H3 loop which is part of LBP), resulting in a large LBP with considerable plasticity that adapts to structurally and chemically diverse molecules [31, 32]. The molecular weight of compounds binding to PXR varies from 200 to more than 800 Da. Rifampicin is the largest known PXR agonist.

Similar to PXR, CAR binds structurally diverse ligands, however the spectrum is less pronounced since the ligand-binding pocket is much smaller and less flexible [33]. Known ligands are 5-androstan-3-ol and 5-androst-16-en-3-ol as well as pregnanedione [34-36]. Also prescription drugs such as artemisinin and meclizine have been identified as CAR ligands, (meclizine acts as inverse agonist) [29,37]. Besides direct activation upon ligand binding, CAR can be also indirectly induced in a yet unknown manner by compounds such as phenobarbital or phenytoin, respectively [38].

\section{Current status of NR drug discovery research}

\subsection{Other nuclear receptors as potential drug targets}

Current NR research not only continues to develop improved modulators for receptors already targeted by approved drugs as described before, but also intends to address other NRs that have been identified to be involved in various diseases with agonists or antagonists. Representative examples for these nuclear receptors are the liver $X$ receptor (LXR), farnesoid $X$ receptor $(\mathrm{FXR})$ or PPARS.

LXR exists in two isoforms: LXR $\alpha$ (NR1H3) and LXR $\beta$ (NR1H2). While LXR $\alpha$ is mainly expressed in the liver, LXR $\beta$ is ubiquitously expressed. Endogenous LXR ligands are oxysterols, oxigenated derivatives of cholesterol (e.g. 27-hydroxycholesterol, cholestenoic acid) and 
cholic acid [39]. Both isoforms are involved in transcriptional control of genes involved in uptake, transport, efflux and excretion of cholesterol in a tissue-dependent manner as well as inflammatory responses in the CNS [40,41]. LXR $\alpha$ and LXR $\beta$ bind as heterodimers (RXR $\alpha$ ) to the response elements of LXR target genes which comprise (among others) ABC transporters, apolipoprotein A and fatty acid synthase. Therefore, compounds modulating LXR are considered as potential therapeutics for metabolic and neurodegenerative diseases. Many small molecules targeting LXR have been identified in the last decades and several have reached clinical phases [42]. A problem of LXR agonists are adverse effects due to LXR $\alpha$ activation in the liver, resulting in increased hepatic lipogenesis, hypertriglyceridemia and liver steatosis. As both isoforms share $77 \%$ sequence homology in DBD and LBD, the identification of selective agonists is not a trivial task. Nevertheless, some selective LXR $\beta$ agonists have been reported. The first identified selective agonists ( $\mathrm{N}$-acylthiadiazolines) activate the $\beta$-isoform several times more efficient than the $\alpha$-isoform [43]. A phenylsulfone-substituted quinoxaline compound has been identified as partial agonist of LXR $\beta$ (potent activator in kidney cells, low induction in liver cells) and revealed poor affinity towards LXR $\alpha$ [44]. Very recently, LXR $\beta$-selective compounds have been identified using pharmacophore modelling and shape-based virtual screening which activated LXR $\beta$ up to 1.8-fold over the $\alpha$-isoform [45].

As described above, two members of the PPAR subfamily are already addressed by approved drugs. Current research also focuses on the third member, PPARס. Expressed in most metabolically active tissues, the receptor regulates expression of a set of genes involved in glucose homeostasis and fatty acid synthesis/storage, mobilization and catabolism [46]. Due to its physiological functions, agonists of PPARS are considered as potential therapeutics of the whole spectrum of metabolic syndromes including diabetes, atherosclerosis and obesity [46]. In addition, PPAR $\delta$ agonists have been shown to stimulate oligodendrocyte differentiation and thus are considered as potential therapeutics in demyelinating disorders such as multiple sclerosis [47].

So far, a large bunch of receptor agonists have been identified that advanced research on this PPAR subtype and its physiological role [48]. Recently, a benzisoxazole has been identified as PPARס-selective agonist with an $\mathrm{EC}_{50}$-value of $4.5 \mathrm{nM}$ as determined using a cell-based assay [49]. Another example is GW501516, a PPARס ligand developed by GlaxoSmithKline that has been identified by combinatorial chemistry and structure-based design [50]. The compound revealed high affinity and potency against PPARS while showing a more than 1000-fold selectivity over PPAR $\alpha$ and PPAR $\gamma$. Despite its favorable effect on the receptor and no toxicity in human trials, the compound was not developed further. Later studies in animal models revealed the compound to possess a substantial carcinogenic potential.

Besides the discovery of receptor-selective compounds, the development of dual agonists activating two PPAR subtypes or pan-agonists activating all members of the PPAR subfamily is also actively pursued. Although a compound of the glitazar class has recently been approved as drug, no further compounds have reached so far the market.

Another example of a promising nuclear receptor drug target is the farnesoid X receptor (FXR) which binds bile acids, the final product of the cholesterol metabolism [51]. Due to the toxic properties of bile acids their levels have to be tightly regulated. FXR can regulate the bile 
homeostasis by activating other nuclear receptors (such as CAR, PXR and VDR) [52], cell surface receptors (G protein-coupled bile acid receptor 1), receptor and calcium-gated potassium channels. FXR signaling is involved in the regulation of intestinal bacterial flora [53], liver regeneration [54] and - in case of misregulation - to hepatocarcinogenesis [55, 56]).

FXR is considered as a suitable drug target for the treatment of dyslipidemia, atherosclerosis and cholestatic disorders, and some effort has been spent on identification and development on agonists [57]. From the already approved drugs, the antiparasitic drug ivermectin has been identified as a FXR agonist [58]. In spite of some side effects related to trygliceride misbalance, FXR agonists are able to recover cholestasis and antidyslipidemic effects [59, 60].

\subsection{Co-activator-binding inhibitors as a future therapeutic approach?}

All currently approved drugs targeting NRs address the ligand-binding pocket. In recent years, novel approaches for inhibiting NRs have emerged in order to overcome limitations of LBPtargeting drugs. Problems may be side effects due to displacement of the natural ligand, thereby disturbing physiological homeostasis, but also limitations of the ligand diversity as imposed by the shape and composition of the LBP as well as resistance of the receptor due to mutations [61].

In order to overcome these limitations, non-LBP pockets have been investigated for their potential to harbour small molecules and thereby modulate receptor activity. In particular, sites involved in NR-co-activator interactions or receptor-DNA contacts have been investigated in detail. The modulation of NR-co-activator interactions has been studied extensively in recent years and several studies have reported the successful discovery of co-activator binding inhibitors (CBI) which confirms the applicability of this approach [62]. Nevertheless, the development of CBIs is challenging due to specificity issues (more than 300 coregulators have been identified in humans so far) as well as the general conformational flexibility of NRs.

Most studies have concentrated on the co-activator binding site (AF-2 site). Several alternate mechanisms have been proposed for explaining the deleterious effects of interference with AF-2. Besides inhibition of co-activator binding , this may involve corepressor recruitment, increase on the NR turnover levels, blockage of the dimer formation, or inhibition of interactions between the N- and C-terminal domain [8].

Because of the anti-androgen resistance phenomenon of prostate cancer [63], modulators addressing the AF-2 site have attracted attention. The effect of AR co-regulator binders is considered to function by inhibition of the N/C interaction that occurs between AF- 1 and AF-2 which is considered as crucial for stabilization of the receptor-ligand complex in the active conformation [61]. Interestingly, AR not only binds co-activators carrying the LxxLL motif but also the more bulky FxxLF motif. X-ray crystal structures of the AR LBD revealed the presence of deep pockets at the AF-2 site, enabling accommodation of the large FxxLF side chains. Not only synthetic peptides, based on a pyrimidine core, were able to selectively displace AR coactivator molecules, as corroborated by FRET assays, and interfere with transcription activation [64], but also small molecules have been identified to disrupt co-activator binding using a virtual screening campaign with subsequent experimental validation (Figure 4B \& D) [65]. 
Another receptor for which AF-2 binders have been identified is the thyroid hormone receptor (TR). TR subtypes are a target for treatment of hyperthyroidism or cardiac arrhythmias. The co-activator binding site of TR $\beta$ has been successfully targeted using macrolactam-constrained co-activator peptides [66]. Another approach to address TR $\beta$ is the use of suicide inhibitors. The proposed pro-drug, DHPPA (or 3-(dibutylamino)-1-(4-hexylphenyl)-propan-1-one)) is able to interact with the AF-2 surface in a similar way as he co-activator SRC-1 [67].

Also the xenosensor PXR has been investigated for inhibition by CBIs. As described before, the receptor has not been addressed for specific treatment. Instead, PXR AF-2 inhibitors are intended to prevent premature drug metabolism, leading to prolonged half-lifes that may result in lower dosages and less side effects. In addition, PXR antagonists may be applied to prevent drug-drug-interactions in patients treated with combination therapies or multimorbid patients exposed to a variety of drugs. Antibiotics such as fluconazole, enilconazole and ketoconazole inhibit PXR, resulting in reduced expression levels of CYP3A4 and MDR1 [68]. It has been shown that the compounds inhibit PXR-SRC-1 interactions by binding to the AF- 2 site using site-directed mutagenesis [69]. Based on the proposed binding mode and the resulting receptor-ligand interactions, a pharmacophore has been generated [70]. In a followup study the pharmacophore has been utilized for the identification of several small molecule antagonists of PXR, including the FDA approved prodrug leflunomide [71].

In addition to the AF-2 site, other regions of the LBD also have been successfully targeted by small molecules that modulate the interaction of the receptor with co-activator proteins. Recently, a small hydrophobic pocket formed by amino acids located on helix 1, the H1-H3 loop as well as helix 9 has been identified on the AR surface (termed BF-3 site) to be addressable by small molecules [67]. By testing a set of approximately 55,000 compounds from various sources using fluorescence polarisation and X-ray crystallographic screenings, several small molecules such as 3,3',5-triiodothyroacetic acid, T3 or flufenamic acid have been identified to bind to BF-3 (Figure 4C \& E) [67]. The BF-3 site is conserved among steroid hormone receptors such as progesterone receptor, mineralocorticoid receptor and glucocorticoid receptor, suggesting that a similar approach could also lead to the identification of CBIs against these receptors [72]. Compounds binding to BF-3 seem to allosterically interfere with co-activator binding to the AF-2 site [73]. In the last years, several studies have reported the successful discovery of additional small molecules targeting the BF-3 pocket. Using virtual screening in combination with biochemical and cell-based tests, a set of structurally diverse AR inhibitors has been identified. Binding to BF-3 has been confirmed by solving the X-ray crystal structure of the receptor-ligand complex. In a follow-up study, one of these molecules was further developed to $\mathrm{AR}$ inhibitors with $\mathrm{IC}_{50}$ values at low micromolar range [74]. Subsequently the crystal structure of the AR in complex with 2-((2-phenoxyethyl)thio)-1H-benzimidazole confirmed molecule binding at the BF-3.

\section{Methods to assess ligand binding and/or activation of nuclear receptors}

In the last thirty years several molecular and cell biology standard methods have been applied to investigate nuclear receptor functions and regulations [75]. For example cDNA cloning has 
been used to identify the genes encoding orphan nuclear receptors. In order to discover hormone-response elements, electrophoretic mobility shift assays (EMSA) and chromatinimmunoprecipitation (ChIP) have been applied as well as different GST pull-down assays [76-78].

To investigate the biological effect of a compound, a variety of binding assays have been developed. A standard ligand-binding how a ligand competes with a known labeled ligand in binding to the receptor [79]. In recent years a variety of non-radioactive activity assays such as biochemical-based fluorescent polarization and time-resolved fluorescence assays have been developed [80]. Detailed analyses of the macromolecular interaction of ligand binding, including affinity- and binding kinetics, have been performed by the surface plasmon resonance (SPR) technology (see also below) [81].

Due to their relevance as therapeutic targets [83] the pharmaceutical industry prioritised the development of novel assay systems that allowed to accelerate the throughput and the screening of large compound collections. Therefore, a couple of academic laboratories as well as pharmaceutical and biotech companies have spent much effort in the development of highthroughput screening compatible screening assays in the last decade [84, 85]. These efforts led to modified methodologies with higher throughput and less variability. A couple of NR screening campaigns have used smal molecule libraries such as Sigma-Aldrich LOPAC, Biomol and Tocris/TimTec bioactive collection and U.S. Food and Drug Administration 1 and 2 collection [86]. Despite the fact that most of the assays have been designed for certain targets the principles could be expanded to any NR, making these assay formats accessible to drug discovery applications.

In the following, a selection of relevant biochemical and cell-based assays as well as in silico methods is presented that is frequently used in NR research, both in academia and pharmaceutical industry.

\subsection{Transactivation assays}

The most common test systems for nuclear receptor activation are cell-based transactivation assays. These assays rely on the potential of nuclear receptors to activate transcription upon ligand binding $[87,88]$. In general, this is achieved by transfection of cells with an expression vector for the receptor and a reporter vector that contains the binding site for the receptor and also encodes for a protein that, when incubated with the appropriate substrate, result in a detectable signal.

Standard protocols involve transient transfection of the receptor and a response elementreporter gene construct [89]. The general advantage of these cell-based assays is that they allow screening of large compound libraries in a reproducible fashion [85]. Until now many cell lines have been described as possible recipients of these vectors, including $\mathrm{CHO}, \mathrm{HuH7}, \mathrm{MCF}-7$, HEK293, HepG2 and Caco-2 cells [90]. Using transient transfection systems a couple of investigators identified activators for various nuclear receptors [62]. 


\subsection{Corregulator-recruitment (mammalian two-hybrid, CARLA)}

An alternative transactivation assay system is the mammalian two-hybrid system. This assay represents a powerful approach for detecting protein-protein interactions in cells, which has evolved from the original two-hybrid system into a method for identifying NR ligands. The system is based on the finding that co-activators and co-repressors are involved in the regulation of NR function. Following ligand binding, many NRs perform a conformational change and form a specific co-activator binding pocket, which permits co-activator binding. In the mammalian two-hybrid approach, chimerical receptors containing the LBD of interest are fused to the DBD of the yeast transcription factor GAL4, which binds to specific NR response elements. The interaction between the NR and its co-activator is detected using a reporter gene containing multiple copies of the GAL4 upstream activating system.

Examples are mammalian two-hybrid assays consisting of the LBD of human CAR and coactivator SRC-1 fused to GAL4 DBD. In this assay the ligand binding enhances the interaction between LBD and SRC-1, which is detected by the reporter gene activity [91]. Using a similar assay a set of agonists and inverse agonist were identified to bind to the human CAR even if some results were contradictory $[92,93]$. It was speculated that the use of truncated chimerical receptors resulted in subtle conformational changes and unspecific protein-protein interactions [90], which led to the conclusion that utilization of full-length receptors is more sensitive and better reflects the in vivo situation [85].

An assay type that allows monitoring of co-activator recruitment is the Co-Activator-dependent Receptor Ligand Assay (CARLA) [100]. CARLA is based on the principle that ligandbinding stimulates interaction between the NR and a co-activator protein which is part of the normal pathway for transcriptional activation. Technically, CARLA is a GST pull-down assay using a GST-receptor fusion protein and a labelled co-activator. The GST fusion protein is immobilized on glutathione-sepharose beads and incubated with the co-activator in the presence or absence of potential ligands. In this setup an actual ligand of the receptor enhances the interaction of the receptor with the co-activator and thereby increases the amount of coactivator that is pulled down. In summary, CARLA is a functional binding assay that reports on the molecular consequence of ligand binding.

Originally, the assay has been developed for the PPARs [94-96]. However, with some modifications the assay format can be used for any nuclear hormone receptor and several coactivators including SRC-1, CBP/p300, Tif2, Rac3, GRIP-1, and RIP140 [97].

\subsection{Surface plasmon resonance, biochemical assay formats, AlphaScreen ${ }^{\circledast}$ and LANCE ${ }^{\circledast}$}

Detailed analyses of the macromolecular interaction of ligand-binding including affinity and binding kinetics is performed by Surface Plasmon Resonance (SPR) [81]. This technology overcomes the common limitations of indirect non-equilibrium methods due to its high sensitivity [82]. In the standard SPR approach, only small amounts of receptor protein are immobilized onto solid phase while different concentrations of the ligand are passed in flow over the surface. In NR research, SPR has been applied to detect and quantify receptor-DNA, receptor-receptor, as well as receptor-ligand interactions [81]. In the past it has also been used 
to characterize binding of co-regulators of a variety of nuclear receptors including thyroid receptor, estrogen and androgen receptor [98-101]. With regard to high-throughput applications, a variety of non-cell based assay formats based on the AlphaScreen ${ }^{\circledR}$ or LANCE $^{\circledR}$ technology have been described [102-104].

AlphaScreen ${ }^{\circledR}$ is a non-radioactive homogeneous proximity assay that relies on energy transfer between an acceptor and a donor bead brought into proximity via biological interaction. The donor beads are embedded with a photosensitizer, which converts oxygen to an excited state upon illumination. If a biomolecular interaction drags an acceptor bead into close proximity of a donor bead, the excited singlet oxygen will transfer its energy to the acceptor bead leading to emission of light depending on the fluorophore in the acceptor beads. Each donor bead is capable of generating up to 60,000 singlet oxygen molecules with a half-life of 0.3 seconds, allowing measurements in a time-resolved mode and with substantial signal amplification. The technology can used to rapidly develop high-throughput screening (HTS) assays for NRs [105-107].

A nuclear receptor AlphaScreen ${ }^{\circledR}$ assay is based on the ligand-activated biomolecular interaction between NR and its co-activator, followed by the detection of this interaction using AlphaScreen ${ }^{\circledR}$ compatible reader technology. For many NRs a consensus co-activator peptide sequence (LxxLL motif) is sufficient for the interaction of the agonist-bound receptor with LBD. The detection can be realised by various strategies depending on the nature of the involved binding partners. Rouleau \& Bossé (2006) described such an AlphaScreen ${ }^{\circledR}$ Assays, for estrogen receptor $\alpha(E R \alpha)$ and retinoic acid receptor $\gamma(\mathrm{RAR} \gamma)$ [107]. Other configurations depending on the availability of respective detection reagents, tags and beads are also possible which have already been described for e.g. FXR receptor [105].

Another well validated assay type for studying NR-ligand interactions is based on the LANCE $^{\circledR}$ Technology [102]. In the LANCE ${ }^{\circledR}$ assay, a signal is generated when a donor molecule labelled with chelate europium $(\mathrm{Eu})$ gets into proximity of the acceptor molecule labelled with allophycocyanin (APC). When a biological interaction brings the donor and the acceptor into close proximity, excitation of the Eu-chelate at $340 \mathrm{~nm}$ allows Fluorescence Resoncance Energy Transfer (FRET) to the acceptor APC molecule resulting in fluorescence emission at $665 \mathrm{~nm}$. Long stakes shift and excited-state lifetimes of Europium complex (hundreds of microseconds) warrant Time-Resolved FRET (TR-FRET) analysis.

In LANCE $^{\circledR}$ nuclear receptor assays the same biomolecular interactions between the ligand binding domain and the NR box are addressed. In principle different binding partners can be used depending on their stability and availability: Examples for combinations are: i) Interaction between agonist-bound receptor or receptor LBD and LxxLL motif-containing peptide and/or ii) interaction between an apo- or holo-receptor and the co-repressor interaction domain.

There are a few examples in literature where LANCE assays based on the interaction between receptor and co-activator-derived peptide have been applied [108-110]. Most of the assays reported involve the interaction of biotinylated LxxLL peptides and a tagged receptor LBD. The complex formation is detected using Eu-labelled antibody and APC-labelled streptavidin. 
A large variety of Lance Eu- and APC-labelled reagents is commercially available which allow the capture of differently tagged receptors and coactivators [111]. A great advantage of applying the LANCE technology is the long signal stability, which can be more than $48 \mathrm{~h}$.

\subsection{Identification of NR modulators using in silico methods}

Besides experimental approaches, computational methods have also been extensively applied in order to identify novel agonists or antagonists. The availability of LBD crystal structures allows employment of structure-based virtual screening techniques, for example molecular docking of virtual compound libraries. If the desired NR structure is not available, homology modeling techniques can be used to obtain structural data. Since the LBD structure is highly conserved this approach often results in high-quality protein models.

Once LBP-bound ligands have been co-crystallized, further methods such as pharmacophorebased searches can be applied that make use of specific protein-ligand interactions. The method is also often used as filtering step to reduce the number of compounds to be docked when applying structure-based virtual screening techniques. In any case, a virtual hit requires experimental investigation for validating its modulating effect on the nuclear receptor.

Many studies have reported the successful application of virtual screening approaches for the identification of NR agonists and antagonists, thereby confirming the suitability of these methods. Besides crystal structure data, also homology models have been utilized for the identification of NR agonists as described for the glucocorticoid receptor (GR) and the constitutive androstane receptor (CAR) that were modeled on the basis of the solved crystal structure of progesterone receptor (GR model) or PXR and VDR (CAR model), respectively $[112,113]$.

\section{Final considerations}

Nuclear receptors are an important protein family involved in many physiological processes. So far, several NRs have been successfully addressed by drugs in order to treat various diseases. Despite significant progress in the understanding of the physiological role of several NRs, the function of many receptors is not well understood which is mainly due to missing information of endogenous ligands. Since only a proportion of receptors are addressed by drugs, there is a tremendous potential for future drug discovery campaigns. The existence of a pronounced ligand-binding pocket renders many receptors addressable to drug-like molecules. The availability of alternative areas addressable by small molecules, for example protein-protein interaction sites on the NR surface, suggests further possibilities for modulating the function of NRs. In order to study NR function and to identify novel receptor modulators, a large set of experimental and computational methods has been developed and successfully applied in many research projects. 


\section{Acknowledgements}

The authors would like to thank Fundação de Amparo à Pesquisa do Estado de São Paulo, FAPESP (grants 2013/10288-1, 2014/50255-8 and 2014/03644-9) as well as Conselho Nacional de Desenvolvimento Científico e Tecnológico, CNPq (grant no 202936/2014-7) for financial support.

\section{Author details}

Thales Kronenberger ${ }^{1}$, Oliver Keminer ${ }^{2}$, Carsten Wrenger ${ }^{1^{*}}$ and Björn Windshügel ${ }^{2 *}$

${ }^{*}$ Address all correspondence to: bjoern.windshuegel@ime.fraunhofer.de or cwrenger@icb.usp.br

1 Unit for Drug Discovery, Department of Parasitology, Institute of Biomedical Sciences, University of São Paulo, São Paulo, Brazil

2 Fraunhofer Institute for Molecular Biology and Applied Ecology IME, Hamburg, Germany

\section{References}

[1] Zhang, Z., et al., Genomic analysis of the nuclear receptor family: new insights into structure, regulation, and evolution from the rat genome. Genome Res, 2004. 14(4): p. 580-90.

[2] Zhou, J. and J.A. Cidlowski, The human glucocorticoid receptor: one gene, multiple proteins and diverse responses. Steroids, 2005. 70(5-7): p. 407-17.

[3] Nuclear Receptors Nomenclature, C., A unified nomenclature system for the nuclear receptor superfamily. Cell, 1999. 97(2): p. 161-3.

[4] Sladek, F.M., What are nuclear receptor ligands? Mol Cell Endocrinol, 2011. 334(1-2): p. 3-13.

[5] Rask-Andersen, M., M.S. Almen, and H.B. Schioth, Trends in the exploitation of novel drug targets. Nat Rev Drug Discov, 2011. 10(8): p. 579-90.

[6] Tora, L., et al., The N-terminal region of the chicken progesterone receptor specifies target gene activation. Nature, 1988. 333(6169): p. 185-8.

[7] Shao, D. and M.A. Lazar, Modulating nuclear receptor function: may the phos be with you. J Clin Invest, 1999. 103(12): p. 1617-8. 
[8] Warnmark, A., et al., Activation functions 1 and 2 of nuclear receptors: molecular strategies for transcriptional activation. Mol Endocrinol, 2003. 17(10): p. 1901-9.

[9] Khorasanizadeh, S. and F. Rastinejad, Nuclear-receptor interactions on DNA-response elements. Trends Biochem Sci, 2001. 26(6): p. 384-90.

[10] Naar, A.M., et al., The orientation and spacing of core DNA-binding motifs dictate selective transcriptional responses to three nuclear receptors. Cell, 1991. 65(7): p. 1267-79.

[11] Umesono, K., et al., Direct repeats as selective response elements for the thyroid hormone, retinoic acid, and vitamin D3 receptors. Cell, 1991. 65(7): p. 1255-66.

[12] Kishimoto, M., et al., Nuclear receptor mediated gene regulation through chromatin remodeling and histone modifications. Endocr J, 2006. 53(2): p. 157-72.

[13] Nolte, R.T., et al., Ligand binding and co-activator assembly of the peroxisome proliferator-activated receptor-gamma. Nature, 1998. 395(6698): p. 137-43.

[14] Greschik, H., et al., Structural and functional evidence for ligand-independent transcriptional activation by the estrogen-related receptor 3. Mol Cell, 2002. 9(2): p. 303-13.

[15] Heery, D.M., et al., A signature motif in transcriptional co-activators mediates binding to nuclear receptors. Nature, 1997. 387(6634): p. 733-6.

[16] Garcia-Becerra, R., et al., Mechanisms of Resistance to Endocrine Therapy in Breast Cancer: Focus on Signaling Pathways, miRNAs and Genetically Based Resistance. Int J Mol Sci, 2012. 14(1): p. 108-45.

[17] Wang, D.Y., et al., Identification of estrogen-responsive genes by complementary deoxyribonucleic acid microarray and characterization of a novel early estrogen-induced gene: EEIG1. Mol Endocrinol, 2004. 18(2): p. 402-11.

[18] Jordan, V.C., Fourteenth Gaddum Memorial Lecture. A current view of tamoxifen for the treatment and prevention of breast cancer. Br J Pharmacol, 1993. 110(2): p. 507-17.

[19] Shafi, A.A., A.E. Yen, and N.L. Weigel, Androgen receptors in hormone-dependent and castration-resistant prostate cancer. Pharmacol Ther, 2013. 140(3): p. 223-38.

[20] Gao, W., C.E. Bohl, and J.T. Dalton, Chemistry and structural biology of androgen receptor. Chem Rev, 2005. 105(9): p. 3352-70.

[21] Helsen, C., et al., Androgen receptor antagonists for prostate cancer therapy. Endocr Relat Cancer, 2014. 21(4): p. T105-18.

[22] Tran, C., et al., Development of a second-generation antiandrogen for treatment of advanced prostate cancer. Science, 2009. 324(5928): p. 787-90.

[23] Saad, F., Evidence for the efficacy of enzalutamide in postchemotherapy metastatic castrate-resistant prostate cancer. Ther Adv Urol, 2013. 5(4): p. 201-10. 
[24] Korpal, M., et al., An F876L mutation in androgen receptor confers genetic and phenotypic resistance to MDV3100 (enzalutamide). Cancer Discov, 2013. 3(9): p. 1030-43.

[25] Li, H., et al., Discovery of Small-Molecule Inhibitors Selectively Targeting the DNABinding Domain of the Human Androgen Receptor. J Med Chem, 2014. 57(15): p. 6458-67.

[26] Dalal, K., et al., Selectively Targeting the DNA Binding Domain of the Androgen Receptor as a Prospective Therapy for Prostate Cancer. J Biol Chem, 2014.

[27] Rakhshandehroo, M., et al., Peroxisome proliferator-activated receptor alpha target genes. PPAR Res, 2010. 2010.

[28] Chang, T.K. and D.J. Waxman, Synthetic drugs and natural products as modulators of constitutive androstane receptor (CAR) and pregnane $X$ receptor (PXR). Drug Metab Rev, 2006. 38(1-2): p. 51-73.

[29] Burk, O., et al., Antimalarial artemisinin drugs induce cytochrome P450 and MDR1 expression by activation of xenosensors pregnane $\mathrm{X}$ receptor and constitutive androstane receptor. Mol Pharmacol, 2005. 67(6): p. 1954-65.

[30] Handschin, C. and U.A. Meyer, Induction of drug metabolism: the role of nuclear receptors. Pharmacol Rev, 2003. 55(4): p. 649-73.

[31] Watkins, R.E., et al., Coactivator binding promotes the specific interaction between ligand and the pregnane X receptor. J Mol Biol, 2003. 331(4): p. 815-28.

[32] Chrencik, J.E., et al., Structural disorder in the complex of human pregnane X receptor and the macrolide antibiotic rifampicin. Mol Endocrinol, 2005. 19(5): p. 1125-34.

[33] Xu, R.X., et al., A structural basis for constitutive activity in the human CAR/RXRalpha heterodimer. Mol Cell, 2004. 16(6): p. 919-28.

[34] Baes, M., et al., A new orphan member of the nuclear hormone receptor superfamily that interacts with a subset of retinoic acid response elements. Mol Cell Biol, 1994. 14(3): p. 1544-52.

[35] Forman, B.M., et al., Unique response pathways are established by allosteric interactions among nuclear hormone receptors. Cell, 1995. 81(4): p. 541-50.

[36] Forman, B.M., et al., Androstane metabolites bind to and deactivate the nuclear receptor CAR-beta. Nature, 1998. 395(6702): p. 612-5.

[37] Huang, W., et al., Meclizine is an agonist ligand for mouse constitutive androstane receptor (CAR) and an inverse agonist for human CAR. Mol Endocrinol, 2004. 18(10): p. 2402-8.

[38] Honkakoski, P., S. Auriola, and M.A. Lang, Distinct induction profiles of three phenobarbital-responsive mouse liver cytochrome P450 isozymes. Biochem Pharmacol, 1992. 43(10): p. 2121-8. 
[39] Yang, C., et al., Sterol intermediates from cholesterol biosynthetic pathway as liver X receptor ligands. J Biol Chem, 2006. 281(38): p. 27816-26.

[40] Hong, C. and P. Tontonoz, Liver X receptors in lipid metabolism: opportunities for drug discovery. Nat Rev Drug Discov, 2014. 13(6): p. 433-44.

[41] Xu, P., et al., LXR agonists: new potential therapeutic drug for neurodegenerative diseases. Mol Neurobiol, 2013. 48(3): p. 715-28.

[42] Loren, J., et al., Liver $X$ receptor modulators: a review of recently patented compounds (2009 - 2012). Expert Opin Ther Pat, 2013. 23(10): p. 1317-35.

[43] Molteni, V., et al., N-Acylthiadiazolines, a new class of liver $\mathrm{X}$ receptor agonists with selectivity for LXRbeta. J Med Chem, 2007. 50(17): p. 4255-9.

[44] Hu, B., et al., Identification of phenylsulfone-substituted quinoxaline (WYE-672) as a tissue selective liver X-receptor (LXR) agonist. J Med Chem, 2010. 53(8): p. 3296-304.

[45] Temml, V., et al., Discovery of new liver $X$ receptor agonists by pharmacophore modeling and shape-based virtual screening. J Chem Inf Model, 2014. 54(2): p. 367-71.

[46] Reilly, S.M. and C.H. Lee, PPAR delta as a therapeutic target in metabolic disease. FEBS Lett, 2008. 582(1): p. 26-31.

[47] Aleshin, S. and G. Reiser, Role of the peroxisome proliferator-activated receptors (PPAR)-alpha, beta/delta and gamma triad in regulation of reactive oxygen species signaling in brain. Biol Chem, 2013. 394(12): p. 1553-70.

[48] Miyachi, H., Design, synthesis, and structure-activity relationship study of peroxisome proliferator-activated receptor (PPAR) delta-selective ligands. Curr Med Chem, 2007. 14(22): p. 2335-43.

[49] Sakuma, S., et al., Synthesis of a novel human PPARdelta selective agonist and its stimulatory effect on oligodendrocyte differentiation. Bioorg Med Chem Lett, 2011. 21(1): p. 240-4.

[50] Oliver, W.R., Jr., et al., A selective peroxisome proliferator-activated receptor delta agonist promotes reverse cholesterol transport. Proc Natl Acad Sci U S A, 2001. 98(9): p. 5306-11.

[51] Huber, R.M., et al., Generation of multiple farnesoid-X-receptor isoforms through the use of alternative promoters. Gene, 2002. 290(1-2): p. 35-43.

[52] Lu, T.T., et al., Molecular basis for feedback regulation of bile acid synthesis by nuclear receptors. Mol Cell, 2000. 6(3): p. 507-15.

[53] Inagaki, T., et al., Regulation of antibacterial defense in the small intestine by the nuclear bile acid receptor. Proc Natl Acad Sci U S A, 2006. 103(10): p. 3920-5.

[54] Huang, W., et al., Nuclear receptor-dependent bile acid signaling is required for normal liver regeneration. Science, 2006. 312(5771): p. 233-6. 
[55] Kim, I., et al., Spontaneous hepatocarcinogenesis in farnesoid $X$ receptor-null mice. Carcinogenesis, 2007. 28(5): p. 940-6.

[56] Gadaleta, R.M., et al., Tissue-specific actions of FXR in metabolism and cancer. Biochim Biophys Acta, 2014.

[57] Pellicciari, R., et al., 6alpha-ethyl-chenodeoxycholic acid (6-ECDCA), a potent and selective FXR agonist endowed with anticholestatic activity. J Med Chem, 2002. 45(17): p. 3569-72.

[58] Jin, L., et al., The antiparasitic drug ivermectin is a novel FXR ligand that regulates metabolism. Nat Commun, 2013. 4.

[59] Huang, H., et al., Discovery and optimization of 1,3,4-trisubstituted-pyrazolone derivatives as novel, potent, and nonsteroidal farnesoid X receptor (FXR) selective antagonists. J Med Chem, 2012. 55(16): p. 7037-53.

[60] Renga, B., et al., Discovery that theonellasterol a marine sponge sterol is a highly selective FXR antagonist that protects against liver injury in cholestasis. PLoS One, 2012. 7(1): p. e30443.

[61] Caboni, L. and D.G. Lloyd, Beyond the ligand-binding pocket: targeting alternate sites in nuclear receptors. Med Res Rev, 2013. 33(5): p. 1081-118.

[62] Abnet, C.C., et al., Transactivation activity of human, zebrafish, and rainbow trout aryl hydrocarbon receptors expressed in COS-7 cells: greater insight into species differences in toxic potency of polychlorinated dibenzo-p-dioxin, dibenzofuran, and biphenyl congeners. Toxicol Appl Pharmacol, 1999. 159(1): p. 41-51.

[63] Chen, Y., C.L. Sawyers, and H.I. Scher, Targeting the androgen receptor pathway in prostate cancer. Curr Opin Pharmacol, 2008. 8(4): p. 440-8.

[64] Gunther, J.R., A.A. Parent, and J.A. Katzenellenbogen, Alternative inhibition of androgen receptor signaling: peptidomimetic pyrimidines as direct androgen receptor/ coactivator disruptors. ACS Chem Biol, 2009. 4(6): p. 435-40.

[65] Axerio-Cilies, P., et al., Inhibitors of androgen receptor activation function-2 (AF2) site identified through virtual screening. J Med Chem, 2011. 54(18): p. 6197-205.

[66] Geistlinger, T.R. and R.K. Guy, An inhibitor of the interaction of thyroid hormone receptor beta and glucocorticoid interacting protein 1. J Am Chem Soc, 2001. 123(7): p. 1525-6.

[67] Estebanez-Perpina, E., et al., Structural insight into the mode of action of a direct inhibitor of coregulator binding to the thyroid hormone receptor. Mol Endocrinol, 2007. 21(12): p. 2919-28.

[68] Huang, H., et al., Inhibition of drug metabolism by blocking the activation of nuclear receptors by ketoconazole. Oncogene, 2007. 26(2): p. 258-68. 
[69] Wang, H., et al., Activated pregnenolone X-receptor is a target for ketoconazole and its analogs. Clin Cancer Res, 2007. 13(8): p. 2488-95.

[70] Ekins, S., et al., Human pregnane $X$ receptor antagonists and agonists define molecular requirements for different binding sites. Mol Pharmacol, 2007. 72(3): p. 592-603.

[71] Ekins, S., et al., Computational discovery of novel low micromolar human pregnane X receptor antagonists. Mol Pharmacol, 2008. 74(3): p. 662-72.

[72] Buzon, V., et al., A conserved surface on the ligand binding domain of nuclear receptors for allosteric control. Mol Cell Endocrinol, 2012. 348(2): p. 394-402.

[73] Grosdidier, S., et al., Allosteric conversation in the androgen receptor ligand-binding domain surfaces. Mol Endocrinol, 2012. 26(7): p. 1078-90.

[74] Munuganti, R.S., et al., Targeting the binding function 3 (BF3) site of the androgen receptor through virtual screening. 2. development of 2-((2-phenoxyethyl) thio)- $1 \mathrm{H}-$ benzimidazole derivatives. J Med Chem, 2013. 56(3): p. 1136-48.

[75] McEwan, I.J., Nuclear Receptors: One Big Familiy, in Methods in Molecular Biology 2009. p. 3-18.

[76] Read, J.T., et al., Receptor-DNA interactions: EMSA and footprinting. Methods Mol Biol, 2009. 505: p. 97-122.

[77] Massie, C.E.a.M., I.G, Chromatin Immunoprecipitation (ChIP) Methodology and Readouts, in Methods in Molecular Biology 2009. p. 123-137.

[78] Goodson, M.L., B. Farboud, and M.L. Privalsky, High throughput analysis of nuclear receptor-cofactor interactions. Methods Mol Biol, 2009. 505: p. 157-69.

[79] Jones, S.A., et al., The pregnane $X$ receptor: a promiscuous xenobiotic receptor that has diverged during evolution. Mol Endocrinol, 2000. 14(1): p. 27-39.

[80] Schulman, I.G. and R.A. Heyman, The flip side: Identifying small molecule regulators of nuclear receptors. Chem Biol, 2004. 11(5): p. 639-46.

[81] Lavery, D.N., Binding affinity and kinetic analysis of nuclear receptor/co-regulator interactions using surface plasmon resonance. Methods Mol Biol, 2009. 505: p. 171-86.

[82] Cheskis, B.J.a.F., L.P., Kinetic analysis of nuclear receptor interactions, in Nuclear Receptors - A Practical Approach, D. Picard, Editor. 1998. p. 95-117.

[83] Pelton, P.D., Nuclear Receptors as Drug Targets, in Minor: Handbook of Assay Development in Drug Discovery, L. K, Editor. 2006, CRC Taylor \& Francis: New York. p. 173-181.

[84] Chu, V., et al., In vitro and in vivo induction of cytochrome p450: a survey of the current practices and recommendations: a pharmaceutical research and manufacturers of america perspective. Drug Metab Dispos, 2009. 37(7): p. 1339-54. 
[85] Raucy, J.L. and J.M. Lasker, Cell-based systems to assess nuclear receptor activation and their use in drug development. Drug Metab Rev, 2013. 45(1): p. 101-9.

[86] Shukla, S.J., et al., Identification of clinically used drugs that activate pregnane $X$ receptors. Drug Metab Dispos, 2011. 39(1): p. 151-9.

[87] Sinz, M., et al., Evaluation of 170 xenobiotics as transactivators of human pregnane $X$ receptor (hPXR) and correlation to known CYP3A4 drug interactions. Curr Drug Metab, 2006. 7(4): p. 375-88.

[88] Dring, A.M., et al., Rational quantitative structure-activity relationship (RQSAR) screen for PXR and CAR isoform-specific nuclear receptor ligands. Chem Biol Interact, 2010. 188(3): p. 512-25.

[89] Willson, T.M. and S.A. Kliewer, PXR, CAR and drug metabolism. Nat Rev Drug Discov, 2002. 1(4): p. 259-66.

[90] Stanley, L.A., et al., PXR and CAR: nuclear receptors which play a pivotal role in drug disposition and chemical toxicity. Drug Metab Rev, 2006. 38(3): p. 515-97.

[91] Auerbach, S.S., et al., Retinoid X receptor-alpha-dependent transactivation by a naturally occurring structural variant of human constitutive androstane receptor (NR1I3). Mol Pharmacol, 2005. 68(5): p. 1239-53.

[92] Moore, L.B., et al., Pregnane X receptor (PXR), constitutive androstane receptor $(\mathrm{CAR})$, and benzoate $\mathrm{X}$ receptor $(\mathrm{BXR})$ define three pharmacologically distinct classes of nuclear receptors. Mol Endocrinol, 2002. 16(5): p. 977-86.

[93] Honkakoski, P., et al., A novel drug-regulated gene expression system based on the nuclear receptor constitutive androstane receptor (CAR). Pharm Res, 2001. 18(2): p. 146-50.

[94] Krey, G., et al., Fatty acids, eicosanoids, and hypolipidemic agents identified as ligands of peroxisome proliferator-activated receptors by coactivator-dependent receptor ligand assay. Mol Endocrinol, 1997. 11(6): p. 779-91.

[95] Inoue, I., et al., Fibrate and statin synergistically increase the transcriptional activities of PPARalpha/RXRalpha and decrease the transactivation of NFkappaB. Biochem Biophys Res Commun, 2002. 290(1): p. 131-9.

[96] Tien, E.S., J.W. Davis, and J.P. Vanden Heuvel, Identification of the CREB-binding protein/p300-interacting protein CITED2 as a peroxisome proliferator-activated receptor alpha coregulator. J Biol Chem, 2004. 279(23): p. 24053-63.

[97] Leo, C. and J.D. Chen, The SRC family of nuclear receptor coactivators. Gene, 2000. 245(1): p. 1-11.

[98] Treuter, E., et al., Competition between Thyroid Hormone Receptor-associated Protein (TRAP) 220 and Transcriptional Intermediary Factor (TIF) 2 for Binding to Nuclear Receptors: IMPLICATIONS FOR THE RECRUITMENT OF TRAP AND p160 
COACTIVATOR COMPLEXES. Journal of Biological Chemistry, 1999. 274(10): p. 6667-6677.

[99] Warnmark, A., et al., The N-terminal regions of estrogen receptor alpha and beta are unstructured in vitro and show different TBP binding properties. J Biol Chem, 2001. 276(49): p. 45939-44.

[100] Shatkina, L., et al., The cochaperone Bag-1L enhances androgen receptor action via interaction with the NH2-terminal region of the receptor. Mol Cell Biol, 2003. 23(20): p. $7189-97$.

[101] Lavery, D.N. and I.J. McEwan, Functional characterization of the native NH2-terminal transactivation domain of the human androgen receptor: binding kinetics for interactions with TFIIF and SRC-1a. Biochemistry, 2008. 47(11): p. 3352-9.

[102] Rouleau, N.H., P., Bossé R. and Hemmilä, I., Development of Nuclear Receptor Homogeneous Assay Using the LanceTM Technology, in Minor: Handbook of Assay Development in Drug Discovery, L. K, Editor. 2006, CRC Taylor \& Francis: New York. p. 209-219.

[103] Rouleau, N.a.B., R., Homogeneous Assay Development for Nuclear Receptor Using AlphaScreenTM Technology, in Handbook of Assay Development in Drug Discovery, L. K, Editor. 2006, CRC Taylor \& Francis: New York. p. 193-207.

[104] Shukla, S.J., et al., Identification of pregnane $X$ receptor ligands using time-resolved fluorescence resonance energy transfer and quantitative high-throughput screening. Assay Drug Dev Technol, 2009. 7(2): p. 143-69.

[105] Glickman, J.F., et al., A comparison of ALPHAScreen, TR-FRET, and TRF as assay methods for FXR nuclear receptors. J Biomol Screen, 2002. 7(1): p. 3-10.

[106] $\mathrm{Wu}, \mathrm{X}$., et al., Comparison of assay technologies for a nuclear receptor assay screen reveals differences in the sets of identified functional antagonists. J Biomol Screen, 2003. 8(4): p. 381-92.

[107] Rouleau, N., et al., Development of a versatile platform for nuclear receptor screening using AlphaScreen. J Biomol Screen, 2003. 8(2): p. 191-7.

[108] Tremblay, G.B., et al., Diethylstilbestrol regulates trophoblast stem cell differentiation as a ligand of orphan nuclear receptor ERR beta. Genes Dev, 2001. 15(7): p. 833-8.

[109] Drake, K.A., et al., Development of a homogeneous, fluorescence resonance energy transfer-based in vitro recruitment assay for peroxisome proliferator-activated receptor delta via selection of active LXXLL coactivator peptides. Anal Biochem, 2002. 304(1): p. 63-9.

[110] Liu, J., et al., A homogeneous in vitro functional assay for estrogen receptors: coactivator recruitment. Mol Endocrinol, 2003. 17(3): p. 346-55. 
[111] Jones, S.A., D.J. Parks, and S.A. Kliewer, Cell-free ligand binding assays for nuclear receptors. Methods Enzymol, 2003. 364: p. 53-71.

[112] Schapira, M., R. Abagyan, and M. Totrov, Nuclear hormone receptor targeted virtual screening. J Med Chem, 2003. 46(14): p. 3045-59.

[113] Jyrkkarinne, J., et al., Insights into ligand-elicited activation of human constitutive androstane receptor based on novel agonists and three-dimensional quantitative structure-activity relationship. J Med Chem, 2008. 51(22): p. 7181-92. 